\title{
FMH und curafutura haben TAR- DOC beim Bundesrat eingereicht
}

\section{Christian Oeschger}

Experte Abteilung Ambulante Versorgung und Tarife

\author{
Nach dreijährigen Verhandlungen haben die FMH und der Krankenversicherungs- \\ verband curafutura Mitte Juli 2019 dem Bundesrat ihren Tarifvorschlag «TARDOC» \\ zur Festsetzung eingereicht. Doch mit der Einreichung ist die Arbeit noch lange \\ nicht getan: Aufgrund der tarifpolitischen Situation wird der Bundesrat aller Vor- \\ aussicht nach ein Vernehmlassungsverfahren durchführen. Ebenso haben die ein- \\ reichenden Tarifpartner und die Genehmigungsbehörde zu vereinbaren, wie eine \\ kostenneutrale Einführung von TARDOC realisiert werden kann. Die FMH hat dazu \\ ein Konzept erarbeitet und dem Bundesrat mit eingereicht.
}

Seit mehr als drei Jahren arbeiten die FMH und ihre beiden Tarifpartner, der Krankenversicherungsverband curafutura (CSS, Helsana, KPT und Sanitas) sowie die Medizinaltarifkommission UVG (MTK), unter dem Dach der gemeinsamen Organisation «ats-tms AG» an der Revision des in die Jahre gekommenen Arzttarifes «TARMED». H+, der Spitalverband, hatte die Gesellschaftsverträge mit der ats-tms AG Ende September 2018 gekündigt und ist per Ende Oktober 2018 aus der ats-tms AG ausgetreten. Mit santésuisse, dem zweiten grossen Krankenkassenverband, konnten sich die Tarifpartner FMH, curafutura und MTK bisher nicht über eine zukünftige Zusammenarbeitsform einigen: Während die der ats-tms AG angeschlossenen Tarifpartner einen weiteren Revisionsstau in Zukunft verhindern möchten und deshalb auf ein Einstimmigkeitsprinzip verzichtet haben, beharrt santésuisse weiterhin auf einem Vetorecht. Da der Revisionsbedarf des TARMED für FMH, curafutura und MTK - erst recht nach dem bundesrätliche Tarifeingriff - unbestritten ist, haben sie sich für die Weiterführung und Finalisierung der seit Jahren andauernden Arbeiten verpflichtet.

\section{Klares Bekenntnis der FMH zur Tarifpartnerschaft}

Die Delegiertenversammlung und die Ärztekammer der FMH haben am 8. und 9. Mai 2019 dem Resultat dieser Arbeit, dem «TARDOC», mit grosser Mehrheit zugestimmt. Mit der Genehmigung von «TARDOC» setzen die Ärztinnen und Ärzte ein starkes Zeichen für einen partnerschaftlichen ambulanten Tarif und zeigen nicht nur Reformwillen, sondern sind auch bereit, den revidierten Tarif umzusetzen. Ebenso hat curafutura Anfang Juli 2019 entschieden, den gemeinsam erarbeiteten Tarif mitzutragen und dem Bundesrat einzureichen. Die MTK ist kein Einreichungspartner, weil der Bundesrat lediglich die Kompetenz hat, den KVG-Tarif festzusetzen. Die MTK wird sich für den Unfall-, Invaliden- und Militärversicherungsbereich jedoch ebenfalls der TARDOC-Variante anschliessen. Die Tarife in den beiden Bereichen sollten sich hinsichtlich der Taxpunkte also nicht mehr unterscheiden.

\section{Festsetzung statt Genehmigung}

Eine Genehmigung durch den Bundesrat kann nur dann erfolgen, wenn eine Mehrheit der Kostenträger am Verhandlungstisch ist. curafutura, die auf Kostenträgerseite verhandelt hat, vertritt aber lediglich ca. 43 Prozent der Versicherten in der Schweiz. Daher haben die FMH und curafutura den Tarif beim Bundesrat zur Festsetzung eingereicht. Im Gegensatz zur Genehmigung muss der Tarifvorschlag in diesem Fall eine dreimonatige Vernehmlassung bei allen Tarifpartnern und interessierten Verbänden durchlaufen. Im Anschluss kann der Bundesrat eigene Anpassungen und Anregungen aus den Vernehmlassungsantworten in den Tarif aufnehmen und diesen voraussichtlich frühestens per 1. Januar 2021 festlegen und für alle Tarifpartner verbindlich in Kraft setzen. 


\section{Kostenneutrale Einführung des TARDOC}

Die Genehmigungsbehörde wird die Tarifstruktur prüfen, also abwägen, ob der Tarif höchstens die transparent ausgewiesenen Kosten der Leistung bzw. höchstens die für eine effiziente Leistungserbringung erforderlichen Kosten deckt. Weiter wird sie die kostenneutrale Einführung sicherstellen müssen. Dazu ist sie gemäss der Verordnung über die Krankenversicherung (Art. 59c Abs. 1 lit. c. KVV) verpflichtet, wonach ein Wechsel des Tarifmodells keine Mehrkosten verursachen darf.

Die Gremien der FMH haben sich im Mai 2019 klar positioniert und unterstützen die kostenneutrale Einführung von TARDOC, die im Grundvertrag festgehalten ist. Bei der kostenneutralen Ablösung des TARMED durch den TARDOC ist der FMH wichtig, dass die nun sorgfältig und ausgewogen verhandelte betriebswirtschaftliche Tarifstruktur intakt bleibt. Fehler sollen aber rasch und jederzeit korrigiert werden können. Änderungen in der Tarifstruktur bleiben also Fehlern und Strukturanpassungen vorbehalten. Die Kostensteuerung hingegen soll gemäss der Methode, welche die FMH dem Bundesrat vorschlägt, über den kantonalen Taxpunktwert bzw. für den spitalambulanten Bereich über die Taxpunktwerte der Spitäler, also sektoriell getrennt, gesteuert werden. Dies hat den grossen Vorteil, dass die Kostensteuerung jederzeit nachkalkulierbar bleibt, während die in sich stimmige betriebswirtschaftliche und gemeinsam verhandelte Struktur nicht tangiert wird.

Konkret stellt sich die FMH die Umsetzung dieser kostenneutralen Überführung des TARMED in den TARDOC folgendermassen vor: In einer 21-monatigen kostenneutralen Überführungsphase soll das Volumen für jede Vertragsgemeinschaft über den spezifischen Taxpunktwert gesteuert werden können. Wo Fehler oder Unstimmigkeiten bestehen, sollen Eingriffe in der Tarifstruktur und an den Abrechnungsregeln erfolgen. Gesteuert und berechnet würden die Anpassungen während dieser Zeit durch ein befristet eingesetztes Kostenneutralitätsbüro. In diesem Gremium

\section{Die Kostenneutralitätsphase}

\section{ist ein Interregnum}

Aktuell befinden sich über 10 Kantone in einem Festsetzungsverfahren. Solange für diese Kantone kein rechtsgültiger Entscheid vorliegt, wird zur Berechnung der Eckwerte des Basisjahrs der tatsächlich abgerechnete Taxpunktwert berücksichtigt. Wird während oder bis zu 5 Jahren nach der kostenneutralen Überführungsphase ein definitiver Entscheid gefällt, so ist mit diesem ein neuer Eckwert zu berechnen und der resultierende Taxpunktwert entsprechend anzupassen. sind die Tarifpartner paritätisch vertreten und werden von weiteren Vertretern seitens der involvierten Stellen (Kantone, Bundesamt für Gesundheit BAG und Preisüberwacher) im Status von Beobachtern begleitet.

\section{Die kostenneutrale Überführungsphase gliedert sich in vier Etappen}

1. Vorbereitung: Für jeden Kanton werden die Starttaxpunktwerte basierend auf einem gemeinsam zwischen den Leistungserbringern und den Kostenträgern vereinbarten Basisjahr festgelegt. Aufgrund des höheren Taxpunktvolumens, das TARDOC gemäss Analysen der FMH mit sich bringen wird, werden die Taxpunktwerte je nach Kanton voraussichtlich um ca. 10-15 Prozent gesenkt. Vorausgesetzt, dass die Transcodierung von der alten auf die neue Tarifstruktur stimmt, müsste mit dieser Taxpunktwertabsenkung derselbe Umsatz erreicht werden wie vor Einführung des neuen Tarifs. Die Herabsetzung des Taxpunktwertes ist nicht als Sparübung ausgelegt. Die Vorgabe ist eine kostenneutrale Überführung.

2. Startphase: Während dieser dreimonatigen Periode werden keine Anpassungen vorgenommen. Sie dient als Einschwingphase für den neuen Tarif Gleichzeitig können aber erste Testläufe für die anschliessende Mess- und Steuerphase durchgeführt werden.

3. Mess- und Steuerphase: In den 12 folgenden Monaten wird einmal im Monat für jeden Kanton der für den Folgemonat gültige Taxpunktwert ermittelt. Messgrösse ist die Entwicklung der Kosten pro Erkrankten bzw. Erkrankte. Die Taxpunktwertanpassungen konvergieren dabei auf einen Zielwert. Sie streben die Stabilisierung der Kosten pro Erkrankte bzw. Erkrankten an, nicht die Kosten pro Monat. Neben den Anpassungen der Taxpunktwerte werden auch allenfalls notwendige Korrekturen in der Tarifstruktur vorgenommen. Darunter fallen unter anderem zusätzliche Abrechnungsregeln, aber auch Anpassungen bei Minutagen oder Spartenkalkulationen.

4. Kompensationsphase: In den letzten 6 Monaten der kostenneutralen Überführungsphase werden die während der Start- sowie Mess- und Steuerphase anfallenden Fehlvolumina in den einzelnen Kantonen über höhere bzw. tiefere, für den Zeitraum der Kompensationsphase beschränkte, Taxpunktwerte abgegolten.

Mit dem Abschluss der letzten Phase sollten sich die Taxpunktwerte eingependelt und die Tarifstruktur 
Korrespondenz: FMH / Abteilung Ambulante Versorgung und Tarife Baslerstrasse 47 CH-4600 Olten Tel. 0313591230 Fax 0313591238 tarife.ambulant[at]fmh.ch ihre Praxistauglichkeit bewiesen haben. Ebenso sollten viele bei der Einführung noch bestehende Fehler in der Struktur korrigiert sein. Vor allem aber - und das erscheint den Expertinnen und Experten wichtig - behält die Struktur so ihre kalkulatorische Basis und ist jederzeit über die zugrundeliegenden Kostenmodelle nachrechenbar und weiterentwicklungsfähig.

\section{curafutura will die Tarifstruktur normieren}

Die FMH und curafutura haben sich beide zur Kostenneutralität bekannt. Sie haben aber unterschiedliche Ansichten über die optimale Methode, mit der diese umgesetzt werden soll. Während die FMH wie beschrieben eine sachgerechte Steuerung über den Preis, also den Taxpunktwert, und nötige Korrekturen der Struktur vorschlägt, zieht curafutura die Steuerung über eine Normierung vor. Dies bedeutet, dass die Tarifstruktur auf dem Level der Taxpunkte (nicht der Taxpunktwerte) mittels eines linearen Faktors abgesenkt und damit gekürzt würde. Die FMH lehnt dieses Vorgehen unter anderem auch deshalb ab, weil die Urabstimmung der FMH im Juni 2016 eine Normierung des damaligen Tarifvorschlags mit fast 90 Prozent abgelehnt hatte.

\section{Vorteile der Steuerung über Struktur und Taxpunktwerte}

Die Methode FMH mit der Sicherung der Kostenneutralität über Taxpunktwerte hat grosse Vorteile in der praktischen Anwendung. Erstens ist die Einführung von neuen Tarifpositionen so wesentlich einfacher zu handhaben als bei einer normierten Struktur.

Ein weiterer grosser Vorteil ergibt sich aus der sektoriellen Korrekturmöglichkeit. Das heisst, mit dem Modell der FMH können der praxisambulante sowie der spitalambulante Bereich separat gesteuert werden. Allfällige unterschiedliche Entwicklungen werden so immer dem verursachenden Sektor angelastet und nicht auf alle Leistungserbringer gleichermassen abgewälzt. Die Normierung würde hingegen zu einer deutlichen Quersubventionierung der Spitäler durch die freie Ärzteschaft führen.

Es obliegt nun dem Bundesrat, die Vorgaben festzulegen, wie die gesetzlich vorgesehene kostenneutrale Überführung von TARMED in den TARDOC umzusetzen ist. Die Erfahrungen aus der Kostenneutralitätsphase bei der Einführung von TARMED zeigen, dass dieses Konzept praxistauglich ist und deutliche Vorteile gegenüber einer linearen Absenkung der Taxpunkte (Normierung) hat.

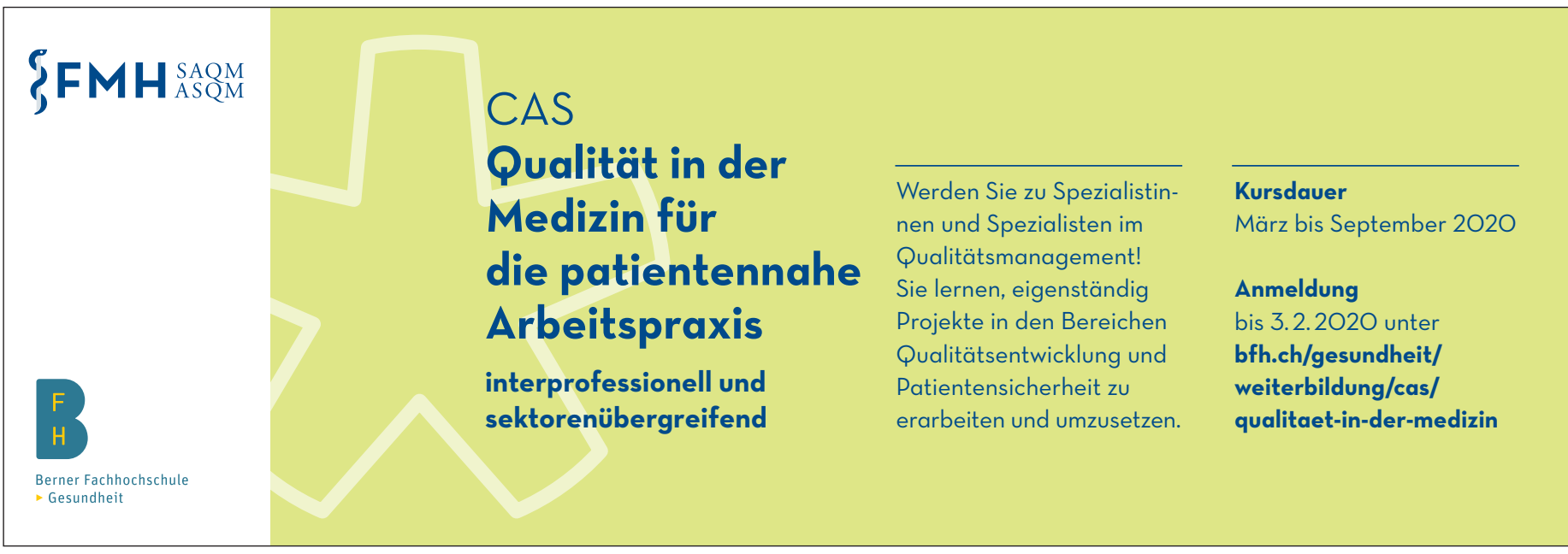

